ARTICLE

https://doi.org/10.1038/s41467-019-12965-1

\title{
In situ formation of mononuclear complexes by reaction-induced atomic dispersion of supported noble metal nanoparticles
}

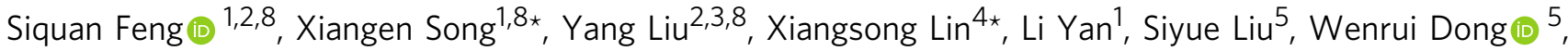
Xueming Yang (1) ${ }^{5,6}$, Zheng Jiang (i) ${ }^{3 \star} \&$ Yunjie Ding (i) ${ }^{1,6,7 *}$

Supported noble metal nanoclusters and single-metal-site catalysts are inclined to aggregate into particles, driven by the high surface-to-volume ratio. Herein, we report a general method to atomically disperse noble metal nanoparticles. The activated carbon supported nanoparticles of $\mathrm{Ru}, \mathrm{Rh}, \mathrm{Pd}, \mathrm{Ag}$, Ir and Pt metals with loading up to 5 wt. \% are completely dispersed by reacting with $\mathrm{CH}_{3} \mathrm{l}$ and $\mathrm{CO}$ mixture. The dispersive process of the Rh nanoparticle is investigated in depth as an example. The in-situ detected l• radicals and $\mathrm{CO}$ molecules are identified to promote the breakage of Rh-Rh bonds and the formation of mononuclear complexes. The isolated Rh mononuclear complexes are immobilized by the oxygen-containing functional groups based on the effective atomic number rule. The method also provides a general strategy for the development of single-metal-site catalysts for other applications.

\footnotetext{
${ }^{1}$ Dalian National Laboratory for Clean Energy, Dalian Institute of Chemical Physics, Chinese Academy of Sciences, 116023 Dalian, China. ${ }^{2}$ University of Chinese Academy of Sciences, 100049 Beijing, China. ${ }^{3}$ Shanghai Synchrotron Radiation Facility, Shanghai Institute of Applied Physics, and shanghai Advanced Research Institute, Chinese Academy of Sciences, 201204 Shanghai, China. ${ }^{4}$ School of Materials and Textile Engineering, Jiaxing University, 314001 Jiaxing, China. ${ }^{5}$ State Key Laboratory of Molecular Reaction Dynamics, Dalian Institute of Chemical Physics, Chinese Academy of Sciences, 116023 Dalian, China. ${ }^{6}$ Hangzhou Institute of Advanced Studies, Zhejiang Normal University, 311231 Hangzhou, China. ${ }^{7}$ State Key Laboratory of catalysis, Dalian Institute of Chemical Physics, Chinese Academy of Sciences, 116023 Dalian, China. ${ }^{8}$ These authors contributed equally: Siquan Feng, Xiangen Song, Yang Liu. *email: xiangensong@dicp.ac.cn; xslin@dicp.ac.cn; jiangzheng@sinap.ac.cn; dyj@dicp.ac.cn
} 
S upported noble metal catalysts have broadly been used in various heterogeneous catalytic reactions. However, deactivation is often an inevitable occurrence ${ }^{1}$. This can be caused by poisoning, coking, and sintering of metal nanoparticles (NPs). The agglomeration of NPs is one of the most important reasons ${ }^{2-4}$. The high ratio of coordinately unsaturated sites and boundaries exposed in small NPs would increase the surface energy and make it more active. Therefore, the driving force for sintering is the thermodynamic instability of small crystallite particles with a high surface-to-volume ratio, and it increases exponentially with the decrease of particle size ${ }^{5,6}$. The strong interaction between the reactants and metal NPs could overcome the interatomic metal-metal bond and facilitate the detachment of mononuclear metal complex from NPs. But the detached unstable monomer would agglomerate and reconstruct into larger particles again to lower their surface energy if there are no ligands to coordinate with the monomer or anchoring site to immobilize the monomer ${ }^{7,8}$. Thus large particles often grow up at the expense of small one by Ostwald ripening process.

Consequently, many approaches to reverse the sintering process by dispersing noble metal NPs have been developed ${ }^{9}$. Some common techniques are oxidation and reduction ${ }^{10,11}$, chlorination, and oxychlorination ${ }^{12,13}$, thermal treatment with halohydrocarbons ${ }^{14-18}$. Dispersion of large particles through the oxidation and reduction or oxychlorination often need high temperature in the range of $773-1073 \mathrm{~K}^{9-13}$. Therefore, these methods require the robustness and chemical inertness of the support. The operating conditions of the thermal treatment with halohydrocarbons are relatively mild. Hardacre et al. demonstrates that large NPs of Au supported on activated carbon $(\mathrm{Au} /$ $\mathrm{AC})$ can be dispersed to single atom, dimer and trimer by $\mathrm{CH}_{3} \mathrm{I}$ during the induction period of vapor methanol carbonylation reaction under $513 \mathrm{~K}$ and 16 bar $^{14}$ or via bromohydrocarbons or iodohydrocarbons treatment at atmospheric pressure in the temperature range of $323-513 \mathrm{~K}^{15}$. In addition, Hardacre et al. also investigate the method of using $\mathrm{CH}_{3} \mathrm{I}$ to disperse $\mathrm{Au} \mathrm{NPs}$ supported on oxide support and discover that there is a drop in average NPs size after the treatment ${ }^{16}$. Thermal treatment with methyl iodide has been proven to be a facile and successful way to redisperse $\mathrm{NPs}^{9}$. However, the dispersion technique is mainly focused on $\mathrm{Au}$ and the improved method may be expanded to other noble metals with high loading.

Although the great progress has been made, the dispersion methods of NPs still face several challenges at present: (i) the operation conditions are usually harsh for many developed methods; (ii) there lacks a universal and simple approach to disperse most noble metal; (iii) most importantly, the degree of dispersion is not high enough for the subsequent process due to the limitation of Ostwald ripening. The atomic dispersion techniques of NPs not only achieves the maximum dispersion of NPs, but also serves as an effective way to fabricate heterogeneous single-metal-site catalysts ${ }^{19-21}$. Heterogeneous mononuclear complex catalyst can serve as a substitute for NPs and homogeneous catalysts, providing an ideal model to understand the catalytic mechanism at the molecular level ${ }^{22}$. A lot of advance works about supported mononuclear complex have been made by Gates et al. and many catalytic reactions are broadly explored by supported mononuclear complex catalyst ${ }^{23-28}$.

Here, we report a general atomic dispersion technique of supported noble metal nanoparticles with loading up to $5 \mathrm{wt} \%$. The nanoparticles of most precious metals supported on activated carbon (AC), such as $\mathrm{Ru}, \mathrm{Rh}, \mathrm{Pd}, \mathrm{Ag}$, Ir, and $\mathrm{Pt}$ are completely atomically dispersed in the form of mononuclear complex by a reaction with a mixture of $\mathrm{CH}_{3} \mathrm{I}$ and $\mathrm{CO}$. The dispersive process is detailedly discussed in the case of Rh metal as an example.

\section{Results}

Discovery of Rh NPs converting to mononuclear complexes. The AC supported $5 \mathrm{wt} . \%$ Rh NPs was first prepared by the calcination of AC supported rhodium chloride in a flow of $\mathrm{N}_{2}$, and then the subsequent reduction by $\mathrm{H}_{2}$ (denoted as $\mathrm{Rh} / \mathrm{AC}$ ). Then the Rh/AC was heated up to $513 \mathrm{~K}$ in a flow of $\mathrm{N}_{2}$, then switched into a flow mixture of $\mathrm{CO}$ and $\mathrm{CH}_{3} \mathrm{I}$ (denoted as $\mathrm{CO} /$ $\mathrm{CH}_{3} \mathrm{I}$ ) and then maintained for $6 \mathrm{~h}$. Then the obtained catalyst was cooled to room temperature in a flow of $\mathrm{N}_{2}$ or $\mathrm{CO}$ for various characterizations (denoted as $\mathrm{Rh}_{1} / \mathrm{AC}$ ). All the experiments were conducted at atmospheric pressure (see Methods).

TEM image and the corresponding particle size distribution (Fig. 1a) show that the size of Rh NPs centered at $4 \mathrm{~nm}$ in the Rh/ $\mathrm{AC}$ catalyst. In the XRD patterns of $\mathrm{Rh} / \mathrm{AC}$ (Supplementary Fig. 1), the peaks at about $41^{\circ}, 47^{\circ}, 70^{\circ}$, and $85^{\circ}$ are attributed to the diffraction of (111), (002), (022), and (113) lattice planes of cubic $\mathrm{Rh}$ particle. The calculated average crystal particle size from XDR patterns is $\sim 5 \mathrm{~nm}$, indicating the presence of a portion of big nanocrystal. After the treatment by $\mathrm{CO} / \mathrm{CH}_{3} \mathrm{I}$, neither appeared peaks belonged to Rh NPs in the XRD patterns (Supplementary Fig. 1), nor could Rh NPs be observed on TEM image (Fig. 1b) over the $\mathrm{Rh}_{1} / \mathrm{AC}$. Furthermore, atomic energy dispersive spectroscopy (EDS) mapping (Supplementary Fig. 2) shows the uniform distribution of $\mathrm{Rh}$ atom on $\mathrm{Rh}_{1} / \mathrm{AC}$. Additionally, isolated $\mathrm{Rh}$ ions or atoms were predominated on the $\mathrm{Rh}_{1} / \mathrm{AC}$ according to the high-angle annular dark-field scanning transmission electron microscopy (HAADF-STEM) images (circled in Fig. 1c). Due to the small difference in atomic number between $\mathrm{I}(Z=53)$ and $\mathrm{Rh}$ $(Z=45)$, the iodine atoms might disturb the recognition of the single Rh atoms in HAADF-STEM. In order to further confirm the sole existence of $\mathrm{Rh}$ single atoms on $\mathrm{Rh}_{1} / \mathrm{AC}$, extended $\mathrm{X}$-ray absorption fine structure (EXAFS) was performed to estimate the average coordination number. EXAFS peaks originally belonged to $\mathrm{Rh}-\mathrm{Rh}(2.69 \AA)$ on $\mathrm{Rh} / \mathrm{AC}$ with a coordination number of 5.0 disappeared, while the fitted coordination numbers of $\mathrm{Rh}-\mathrm{Rh}$ shells were close to zero on $\mathrm{Rh}_{1} / \mathrm{AC}$. As is shown in the fitting result (Fig. 1d, and Supplementary Fig. 3 and Supplementary Table 1), the bond length of Rh-Rh is very close to that of $\mathrm{Rh}-\mathrm{I}$, which makes it difficult to distinguish the two bonds in $R$ space. However, wavelet transform (WT) analysis can provide more accurate and full-scale information for separating backscattering atoms in both radial distance and $k$-space resolution ${ }^{29,30}$. From the WT contour plots of $\mathrm{Rh} / \mathrm{AC}$ and $\mathrm{Rh}_{1} / \mathrm{AC}$ in Supplementary Fig. 4, it can be clearly seen that although the $R$ values of the maximum intensity are quite similar, the $k$ values of $8.1 \AA^{-1}$ for $\mathrm{Rh} / \mathrm{AC}$ and $10.9 \AA^{-1}$ for $\mathrm{Rh}_{1} / \mathrm{AC}$ can be assigned to $\mathrm{Rh}-\mathrm{Rh}$ and $\mathrm{Rh}-\mathrm{I}$ coordination shell, respectively. No signal of $\mathrm{Rh}-\mathrm{Rh}$ backscattering in $k$ space can be observed in $\mathrm{Rh}_{1} / \mathrm{AC}$, which further confirms the complete transformation of Rh NPs into Rh single-atom sites. Three peaks at 2.67, 2.09, and $1.88 \AA$ were fitted to $\mathrm{Rh}-\mathrm{I}, \mathrm{Rh}-\mathrm{O}-\mathrm{AC}$ (oxygen-containing functional group on the surface of the $\mathrm{AC}$ ) and $\mathrm{Rh}-\mathrm{CO}$ distance with a coordination number of 3.7, 1.0, and 1.7, respectively (Fig. 1d, Supplementary Fig. 3 and Supplementary Table 1). The observed coordination number of $\mathrm{Rh}-\mathrm{CO}$ in $\mathrm{Rh}_{1} / \mathrm{AC}$ was roughly consistent with the $\mathrm{CO} / \mathrm{Rh}$ molar ratio of 1.36 which was obtained by $\mathrm{CO}$ temperature-programmed desorption (Supplementary Fig. 5). The attenuated total reflectance-Fourier transform infrared (ATR-FTIR) spectroscopy (Supplementary Fig. 6) shows the peaks attributed to $\left[\mathrm{Rh}(\mathrm{CO}) \mathrm{I}_{4}\right]$ at ca. $2160 \mathrm{~cm}^{-1}$ and $\left[\mathrm{Rh}(\mathrm{CO})_{2} \mathrm{I}_{3}\right]$ at ca. 2030 and $2017 \mathrm{~cm}^{-1}$, respectively ${ }^{31}$. X-ray photoelectron spectroscopy (XPS) analysis (Supplementary Fig. 7 and Supplementary Table 2) shows dominant $\mathrm{Rh}^{3+}$ species existed in $\mathrm{Rh}_{1} /$ AC. $\mathrm{CO}(\mathrm{m} / \mathrm{z}=28), \mathrm{Rh}(\mathrm{m} / \mathrm{z}=102.9)$ and $\mathrm{I}^{\bullet}(\mathrm{m} / \mathrm{z}=127)$ species were detected by laser desorption ionization/time of flight mass spectrometer (LDI/TOF-MS) on $\mathrm{Rh}_{1} / \mathrm{AC}$ (Supplementary Fig. 8). 
a

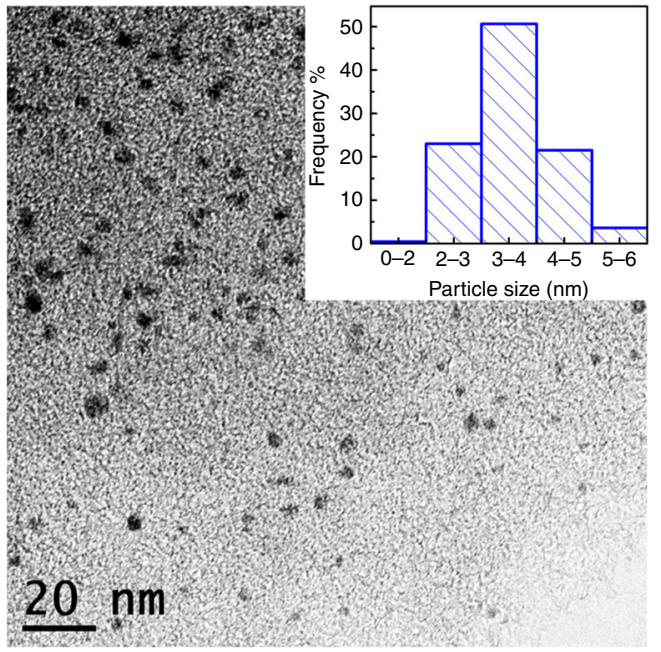

C

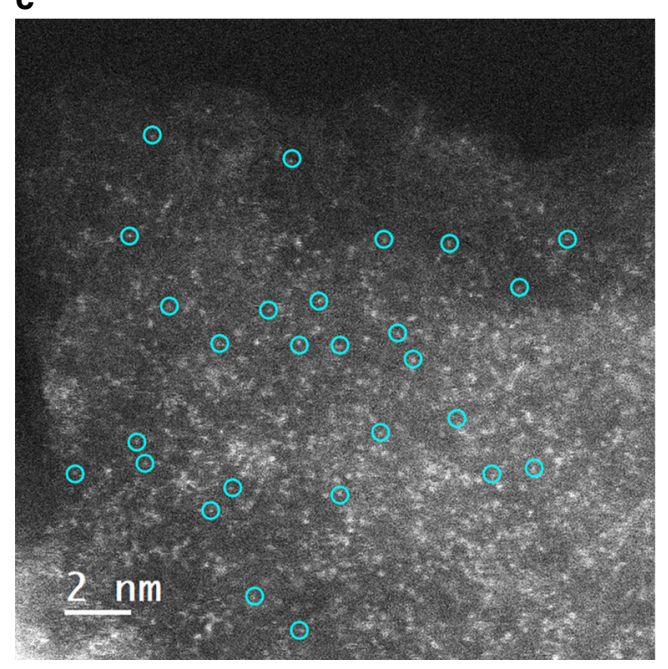

b

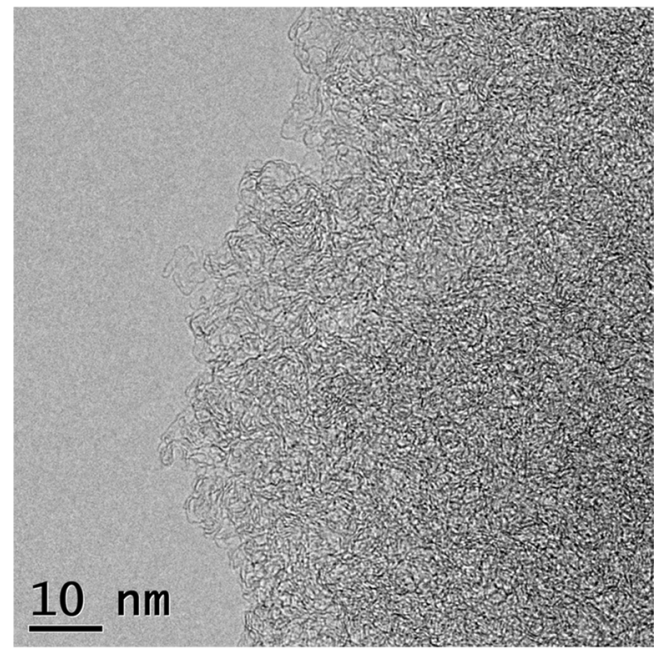

d

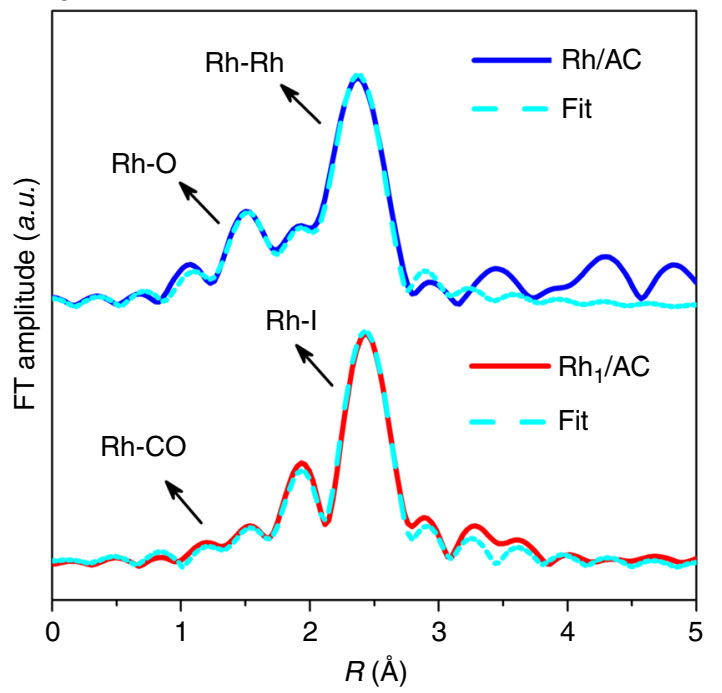

Fig. 1 Structure of the $\mathrm{Rh} / \mathrm{AC}$ and $\mathrm{Rh} / \mathrm{AC}$ catalysts. a The HRTEM image of Rh/AC and the corresponding particle size distribution. $\mathbf{b}$ The HRTEM and c HAADF-STEM image of $\mathrm{Rh}_{1} / \mathrm{AC}$. d Comparison of the experimental (solid line) and fitted (dashed line) curves of $k^{2}$-weight EXAFS spectra of Rh/AC and $\mathrm{Rh}_{1} / \mathrm{AC}$ catalysts

Based on these observations, we can conclude that the atomic dispersion $\mathrm{Rh}$ exists as the form of dominating $\mathrm{Rh}$ $(\mathrm{CO})_{2} \mathrm{I}_{3}(\mathrm{O}-\mathrm{AC})$ and a portion of $\mathrm{Rh}(\mathrm{CO}) \mathrm{I}_{4}(\mathrm{O}-\mathrm{AC})$ in $\mathrm{Rh}_{1} / \mathrm{AC}$. Using our approaches, little metal loss was detected in the process, confirmed by inductively coupled plasma optical emission spectrometer (ICP-OES) analysis (Supplementary Table 3). In addition, this method is simple for large-scale operation and tens of kilograms of $\mathrm{Rh} / \mathrm{AC}$ have been easily atomically dispersed in our laboratory.

Atomically dispersive process and mechanism of Rh NPs. To investigate the $\mathrm{Rh}$ atomically dispersive process, we treated the $\mathrm{Rh} / \mathrm{AC}$ sample with $\mathrm{CO} / \mathrm{CH}_{3} \mathrm{I}$ mixture for different time durations. The HAADF-STEM image of 2 min time on stream (TOS) displayed NP 4-5 nm (Fig. 2a). After $5 \mathrm{~min}$, sub-NP with single atoms scattered around still existed (Fig. 2b), consistent with the corresponding atomic EDS mapping (Fig. 2c). Multimers, trimers and dimers account for the majority after $15 \mathrm{~min}$ treatment (Fig. 2d). These images show that the size of NPs decreased little by little. The quantitative analysis of EXAFS data shows that the coordination number of $\mathrm{Rh}-\mathrm{Rh}$ decreased from 5.0 to $0.7,0.3$ and
0 after 2, 5, and 15 min treatment, individually (Fig. 3a, Supplementary Fig. 9 and Supplementary Table 4). On the contrary, the coordination number between $\mathrm{Rh}$ and $\mathrm{I}$ increased from 0 to 3.3, 3.3 and 3.6 with 2, 5, and 15 min TOS, respectively, and then kept at $~ 3.6$. The variation of $\mathrm{Rh}-\mathrm{CO}$ with TOS was similar to that of $\mathrm{Rh}-\mathrm{I}$. The similar trends could be observed in XRD experiment (Supplementary Fig. 10). The diffraction peaks associated with cubic-Rh NPs decreased sharply over a period of $2 \mathrm{~min}$ and only little features associated with cubic-Rh were found after $5 \mathrm{~min}$. After reaction for $15 \mathrm{~min}$, the diffraction peaks belonged to cubic$\mathrm{Rh}$ completely disappeared. These results suggested the gradual shrinkage of the large Rh NPs due to the continued removal of the exfoliated monomer by $\mathrm{CO}$ on the surface of AC support.

In theory, the cleavage reaction of $\mathrm{CH}_{3} \mathrm{I}$ could occur on the surface of the metal, yielding a large amount of free radicals ${ }^{32}$. The signals of methyl radical $\left(\mathrm{CH}_{3}{ }^{\circ}\right)$ and iodine radicals (I.) can be detected if the quenching of short-lived radicals can be retarded at ultralow pressure or by the high dilution of the feed gas. The short-lived radicals were also observed in the in-situ LDI/TOF-MS experiment when $\mathrm{CO} / \mathrm{CH}_{3} \mathrm{I}$ passed through the $\mathrm{Rh} /$ AC. As shown in Fig. $3 \mathrm{~b}, \mathrm{CH}_{3} \cdot(\mathrm{m} / \mathrm{z}=15)$ and $\mathrm{I}^{\bullet}(\mathrm{m} / \mathrm{z}=127)$ radicals accompanied with $\mathrm{CO}(\mathrm{m} / \mathrm{z}=28)$ and $\mathrm{CH}_{3} \mathrm{I}(\mathrm{m} / \mathrm{z}=142)$ 
a

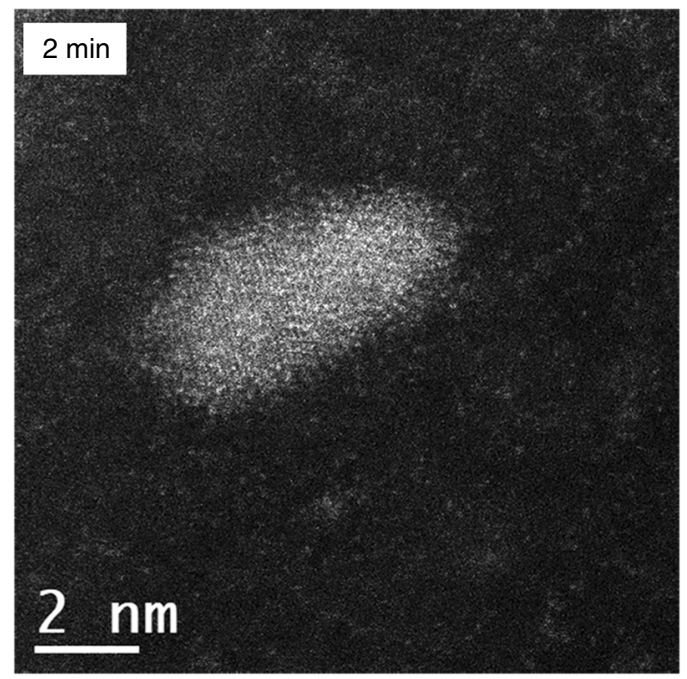

C

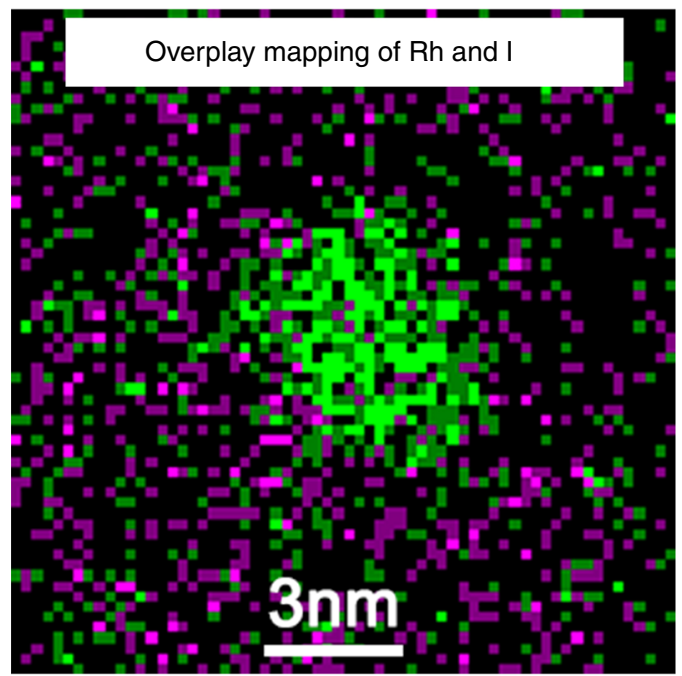

b

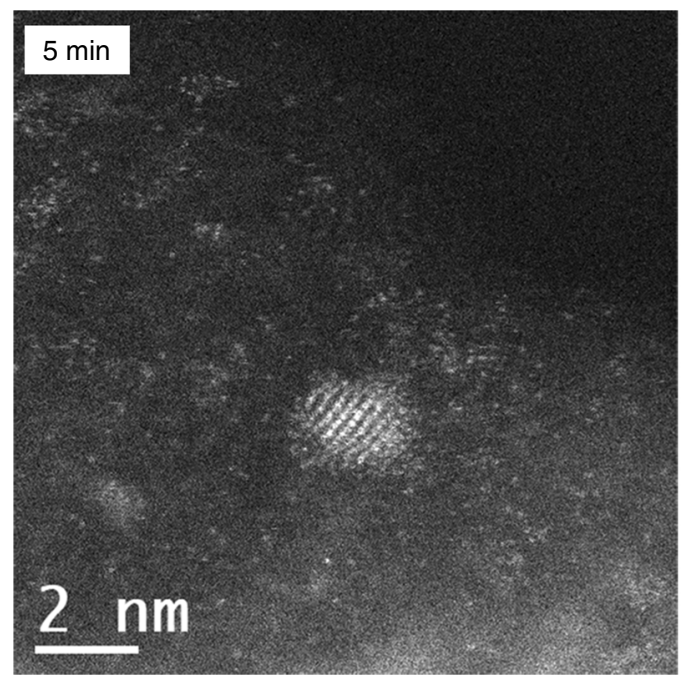

d

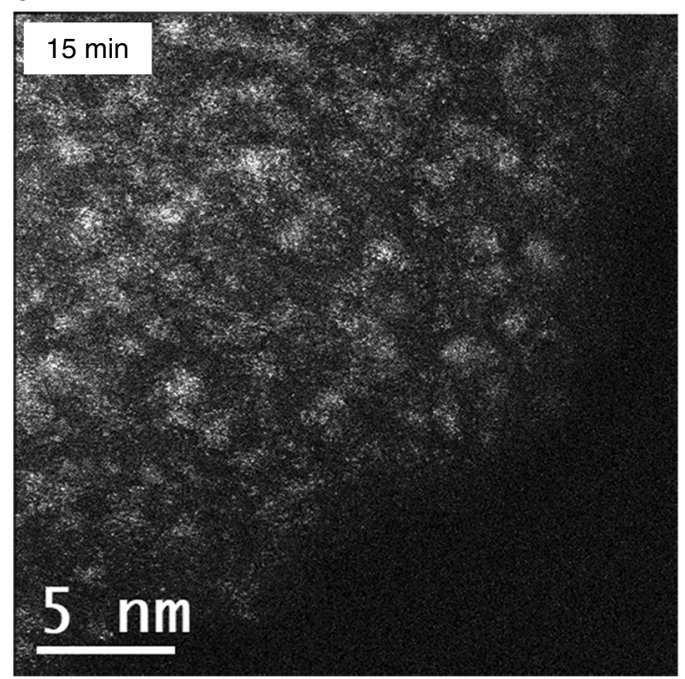

Fig. 2 Time resolution HAADF-STEM pictures of Rh/AC dispersion. The sample treated by $\mathrm{CO} / \mathrm{CH}_{3} \mathrm{l}$ at $513 \mathrm{~K}$ for a $2 \mathrm{~min}, \mathbf{b} 5 \mathrm{~min}$, and $\mathbf{c}$ the corresponding $\mathrm{Rh}$ and I atomic EDS mapping of Fig. $2 \mathbf{b}$, d, 15 min. Green color denotes Rh element and pink color denotes I element

were clearly distinguished in the spectra, implying the homolytic cleavage of $\mathrm{CH}_{3} \mathrm{I}$ on the surface of Rh NPs. While the signals of $\mathrm{Rh}$ carbonyl species could not be found, indicating that the migration of the $\mathrm{Rh}$ carbonyl species occurred on the surface of $\mathrm{AC}^{7}$. In general, high temperature favors the dissociation of $\mathrm{CH}_{3} \mathrm{I}$. However, when the dispersion of NPs started at certain temperature in the form of mononuclear complexes, I• radicals would be continuously consumed and the number of metal NPs also decreased. Therefore, the signal intensity of I• radicals would show a maximum point with the increase of temperature. In fact, the signal variation with temperature (inset of Fig. $3 \mathrm{~b}$ ) shows that the intensity of $\mathrm{I}^{\bullet}$ radicals reached maximum at $438 \mathrm{~K}$ and then decreased quickly from $438 \mathrm{~K}$ to $513 \mathrm{~K}$, suggesting the crucial role of $\mathrm{I}^{\bullet}$ radical in the cleavage of $\mathrm{Rh}-\mathrm{Rh}$ bond and the formation of mononuclear complexes. Further experiment confirmed that the NPs on Rh/AC could also be atomically dispersed at ca. $438 \mathrm{~K}$ when both $\mathrm{CO}$ and $\mathrm{CH}_{3} \mathrm{I}$ existed (Supplementary Fig. 11a), but failed at $373 \mathrm{~K}$ (Supplementary Fig. 11b).

In addition, the similar particle size distribution remained on $\mathrm{Rh} / \mathrm{AC}$ catalyst after individual treatment by CO (Supplementary Fig. 1 and Supplementary Fig. 12a) or $\mathrm{CH}_{3} \mathrm{I} / \mathrm{N}_{2}$ (Supplementary Fig. 1 and Supplementary Fig. 12b) at $513 \mathrm{~K}$ for $6 \mathrm{~h}$, indicating that the sole action of $\mathrm{CO}$ or $\mathrm{CH}_{3} \mathrm{I}$ could not atomically disperse $\mathrm{Rh}$ NP. During the process of Ostwald ripening, the detached monomer would agglomerate into larger particles if there were no anchored site to immobilize the monomer. In order to explore the effect of $\mathrm{O}-\mathrm{AC}$ for the atomic dispersion, the $\mathrm{AC}$ samples were treated under $\mathrm{H}_{2}$ atmosphere at $1273 \mathrm{~K}$ to remove O-AC. After treated with $\mathrm{H}_{2}$, the NMR peaks at 216 and $181 \mathrm{ppm}$ belonged to carbonyl and the peak at $121 \mathrm{ppm}$ ascribed to benzene ring all disappeared, indicating the elimination of almost all oxygencontaining functional groups (Supplementary Fig. 13). The TPDMS further indicates that no O-AC decomposed during the temperature-rise period on the $\mathrm{H}_{2}$ treated AC (Supplementary Fig. 14). Without these oxygen-containing functional groups, the $\mathrm{Rh}$ NPs became even bigger after treated by $\mathrm{CO} / \mathrm{CH}_{3} \mathrm{I}$, which followed Ostwald ripening 33,34 (Supplementary Fig. 15). This indicated the crucial role of $\mathrm{O}-\mathrm{AC}$ for the atomic dispersion of $\mathrm{Rh}$ NPs. Accordingly, the combined actions of proper temperature, $\mathrm{CO}, \mathrm{CH}_{3} \mathrm{I}$, and $\mathrm{O}-\mathrm{AC}$ are indispensable for the atomic dispersion of Rh NPs.

Various oxygen-containing functional groups can be found on the surface of AC, such as carboxyl, lactone, anhydride, phenol, carbonyl, and ether ${ }^{35}$. Carboxyl, lactone, and anhydride are 

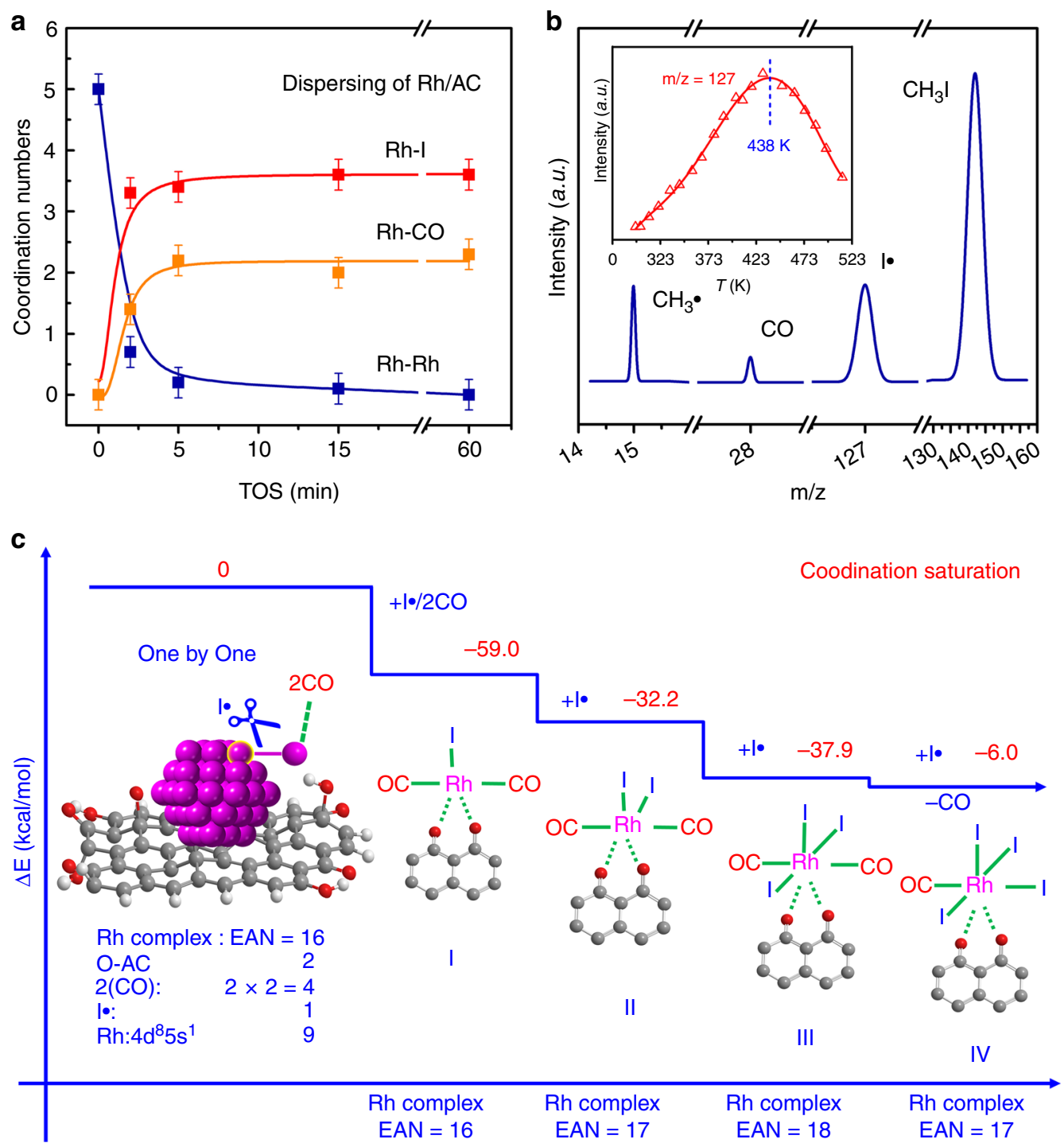

Fig. 3 Structure and model for the atomically dispersive process of Rh NPs. a Coordination number variation of Rh-Rh, Rh-l, and Rh-CO during atomically dispersion process derived from EXAFS result (Supplementary Table 4) of Rh/AC treated with $\mathrm{CO} / \mathrm{CH}_{3} \mathrm{l}$ at $513 \mathrm{~K}$ for different time. $\mathbf{b}$ The representative mass spectra of different reactant and radical species detected during the in-situ reaction between Rh/AC and CO/CH 3 at 513 K by LDI/TOF-MS. The inset is the variation of iodine radicals $\left({ }^{\bullet}\right)$ signals during the reaction between $\mathrm{Rh} / \mathrm{AC}$ and $\mathrm{CO} / \mathrm{CH}_{3} \mathrm{l}$ at different temperature. $\mathbf{c}$ The atomic dispersion model of Rh NPs via one-by-one mechanism based on the 16-18 effective atomic number (EAN) rule

unstable and decompose at low temperature in the form of $\mathrm{CO}_{2}$ (Supplementary Fig. 14). While the phenol, carbonyl, and ether are relatively stable. Interestingly, a carbon vacancy along with either isolated phenol group or adjacent phenol-carbonyl pairs in the reduced graphene oxide surface served as anchored site for single $\mathrm{Pd}$ atom by atomic layer deposition ${ }^{36,37}$. In the $\mathrm{AC}$ supported single sites catalyst for heterogeneous carbonylation, a broken ether bond was proposed as the site to immobilize the single metal ion ${ }^{38}$. For the modeling of DFT calculation, we chose carbonyl and ether as the anchored site for mononuclear complex (Supplementary Fig. 16) based on the key role of carbonyl and benzene groups on the surface of $\mathrm{AC}$ on the formation of mononuclear complexes.

Density function theory (DFT) calculations showed that the atomic dispersion of Rh NPs was accomplished via one-by-one mechanism based on the effective atomic number (EAN) rule $^{39}$ (Fig. $3 \mathrm{c}$ and Supplementary Fig. 17a). Once one atom of Rh NPs was attacked by $\mathrm{CO}$ and $\mathrm{I} \bullet$ free radical (Fig. $3 \mathrm{c}$ and Supplementary Table 5), the $\mathrm{Rh}$ atom was extracted as $\mathrm{Rh}$
$(\mathrm{CO})_{2} \mathrm{I}(\mathrm{O}-\mathrm{AC})$ complex from the metal NPs with a lower system energy of $-59.0 \mathrm{kcal} / \mathrm{mol}$. Furthermore, the dispersion process of $\mathrm{Rh}$ NPs to take the interactions between $\mathrm{Rh}$ and $\mathrm{CO} / \mathrm{I}$ • free radical is spontaneous without transition state (Supplementary Fig. 17b). However, the structure of $\mathrm{Rh}(\mathrm{CO})_{2} \mathrm{I}(\mathrm{O}-\mathrm{AC})$ complex was not the most stable one. The dynamic simulation of $\mathrm{Rh}$ $(\mathrm{CO})_{2} \mathrm{I}(\mathrm{O}-\mathrm{AC})$ attacked by $\mathrm{CO}$ shows that the formation of $\mathrm{Rh}$ (CO) ${ }_{3} \mathrm{I}$ would bring about the breakage of the bond between $\mathrm{Rh}$ and O-AC (Fig. 4a, b). On the contrary, after attacked by I• free radical, the anchoring of $\mathrm{Rh}(\mathrm{CO})_{2} \mathrm{I}_{2}$ on the neighboring oxygen site is favorable, based on the change of both energy and bond distance (Fig. 4c, d). These results manifested that the formation of $\mathrm{Rh}(\mathrm{CO})_{2} \mathrm{I}_{2}(\mathrm{O}-\mathrm{AC})$ was easier than that of $\mathrm{Rh}(\mathrm{CO})_{3} \mathrm{I}(\mathrm{O}-\mathrm{AC})$. Furthermore, the I• free radical could further attack the virtual $\pi^{*}$ orbital of $\mathrm{Rh}$ atom to form more stable $\mathrm{Rh}(\mathrm{CO})_{2} \mathrm{I}_{3}(\mathrm{O}-\mathrm{AC})$ structure or even substitute a $\mathrm{CO}$ molecule to form $\mathrm{Rh}(\mathrm{CO})$ $\mathrm{I}_{4}(\mathrm{O}-\mathrm{AC})$ via the $\mathrm{Rh}(\mathrm{CO})_{2} \mathrm{I}_{2}(\mathrm{O}-\mathrm{AC})$ intermediate with the reactions enthalpies of -70.1 and $-76.1 \mathrm{kcal} / \mathrm{mol}$, respectively (Fig. $3 \mathrm{c}$ and Supplementary Fig. 18). Accordingly, the atomic 

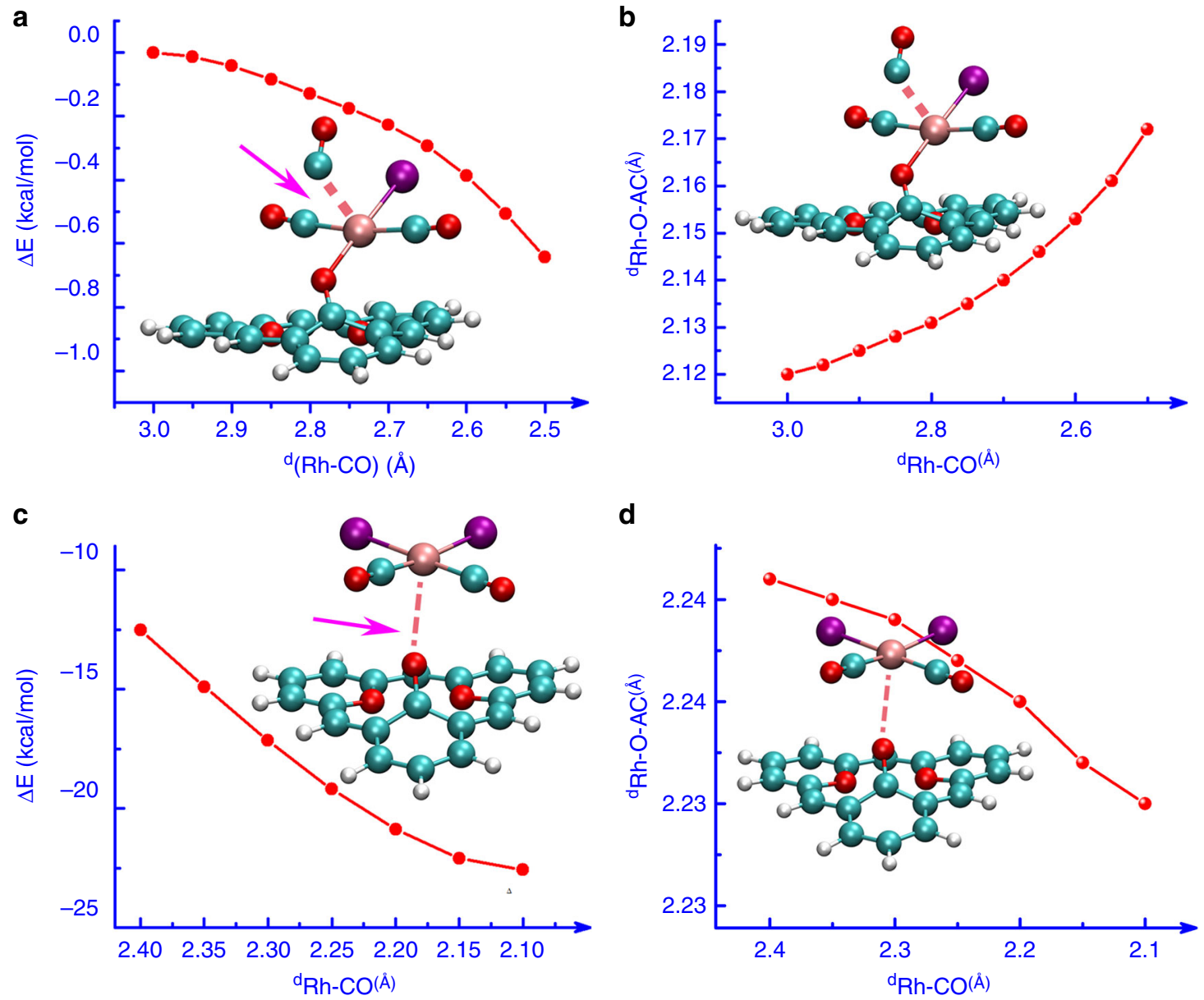

Fig. 4 Dynamic simulation of $\mathrm{Rh}(\mathrm{CO})_{2} \mathrm{I}(\mathrm{O}-\mathrm{AC})$ migration and transformation. $\mathbf{a}$, $\mathbf{b}$ Variation of energy and Rh-O-AC bond distance during the process of $\mathrm{Rh}(\mathrm{CO})_{2} \mathrm{I}(\mathrm{O}-\mathrm{AC})$ attacked by $\mathrm{CO}$. c, d Variation of energy and Rh-O-AC bond distance during the process of $\mathrm{Rh}(\mathrm{CO})_{2} \mathrm{I}_{2}$ anchoring on the neighboring oxygen site in the surface of $A C$

dispersion of AC supported Rh NPs is favorable in thermodynamics when both $\mathrm{CO}$ and $\mathrm{CH}_{3} \mathrm{I}$ participate concurrently in the reaction due to the strong exothermicity.

Universality of atomic dispersion for noble metal NPs. Inspired by the phenomenon, we wonder whether the strategy of atomic dispersion was also applicable for other noble metals. Surprisingly, the NPs of $\mathrm{Ru}, \mathrm{Pd}, \mathrm{Ag}$, Ir, and Pt all could be dispersed to the corresponding single-metal-sites after the treatment of $\mathrm{CO} /$ $\mathrm{CH}_{3} \mathrm{I}$. The TEM and HAADF-STEM pictures in Fig. 5a, b, d, e show that the NPs of Ir and Pt were also successfully dispersed to single atom. The EXAFS spectra after dispersion indicated that the Ir-Ir bonds in Ir NPs and the Pt-Pt bonds of Pt NPs both disappear (Fig. 5c, f and Supplementary Table 6). The singlemetal-site of $\mathrm{Ir}_{1} / \mathrm{AC}$ and $\mathrm{Pt}_{1} / \mathrm{AC}$ also exist in the form of mononuclear complex, similar to that of $\mathrm{Rh}_{1} / \mathrm{AC}$. Similar phenomenon was observed on the Ru/AC (Supplementary Fig. 19), Pd/AC (Supplementary Fig. 20) and Ag/AC (Supplementary Fig. 21) samples after the $\mathrm{CO} / \mathrm{CH}_{3} \mathrm{I}$ similar thermal treatment. Almost all the noble metal NPs could have underwent reverse agglomeration processes and dispersed into the isolated mononuclear complexes in the presence of $\mathrm{CH}_{3} \mathrm{I} / \mathrm{CO}$ mixture. These results demonstrated that the atomic dispersion of supported noble metal nanoparticles was not an individual phenomenon but a shared route to disintegrate metal NPs to single-metal-sites.

\section{Discussion}

A simple and quick protocol for the atomic dispersion of precious metal NPs has been developed. In the case of Rh metal, the combined action of a certain temperature and presence of $\mathrm{CO}$, $\mathrm{CH}_{3} \mathrm{I}$, and $\mathrm{O}-\mathrm{AC}$ were indispensable for the atomic dispersion of Rh NPs. This universal approach could be applicable for most noble metals and also easy for large-scale production. The desired dispersion of NPs can also be further obtained by controlling the post processing conditions of the supported mononuclear complexes $^{25}$, achieving the goal of catalyst regeneration. Furthermore, supported mononuclear complexes with the maximized atom efficiency up to $100 \%$ exhibited unique catalytic performance ${ }^{22}$. The simplicity and uniformity of supported mononuclear complexes offer the advantage of structure determination of active sites dispensable of ultrahigh vacuum. The composition identification of surface metal complex, including ligands and metalsupport bond facilitates the understanding of electron-donor effect for the catalyst reactivity and selectivity, leading to the deep comprehension of structure-performance correlations. Different from many reported fabrication method for the supported mononuclear complexes in which the catalytic active sites were prepared via the reactions of organometallic precursors with the support surface, this work provides a universal and simple strategy to synthesize supported mononuclear complexes of most precious metal. It will bring about new insights and points for the catalyst design. 
a

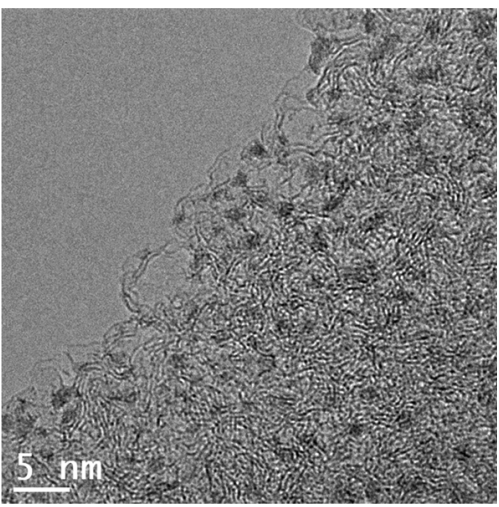

d

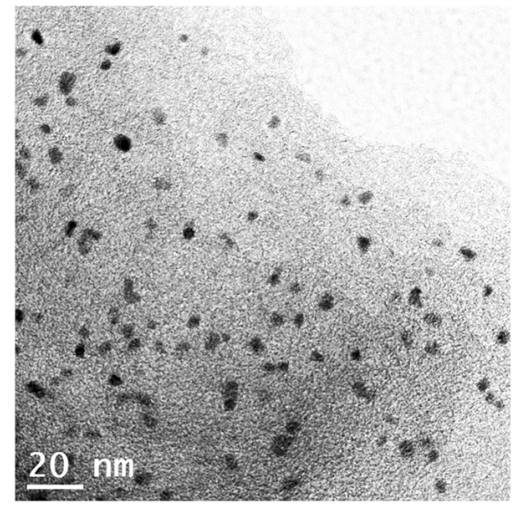

b

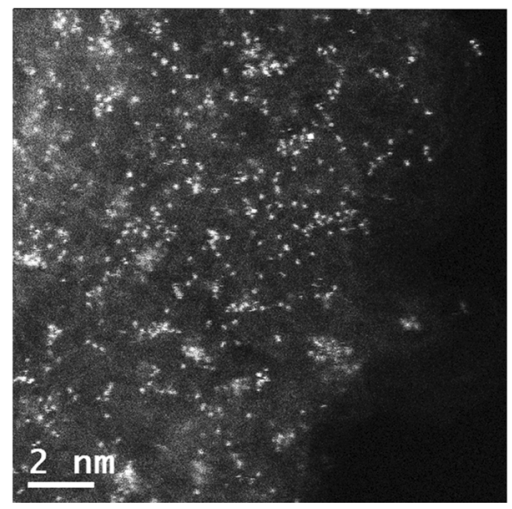

e

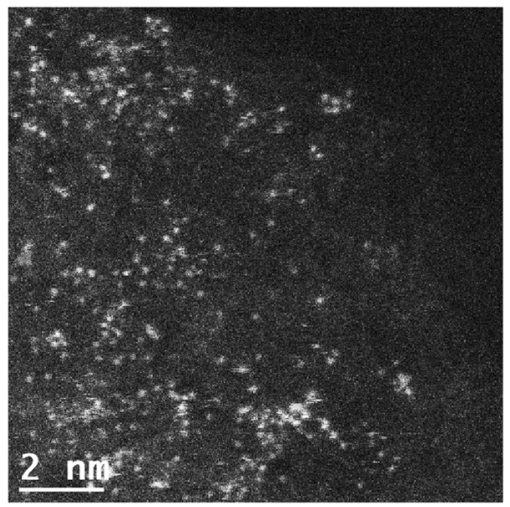

C

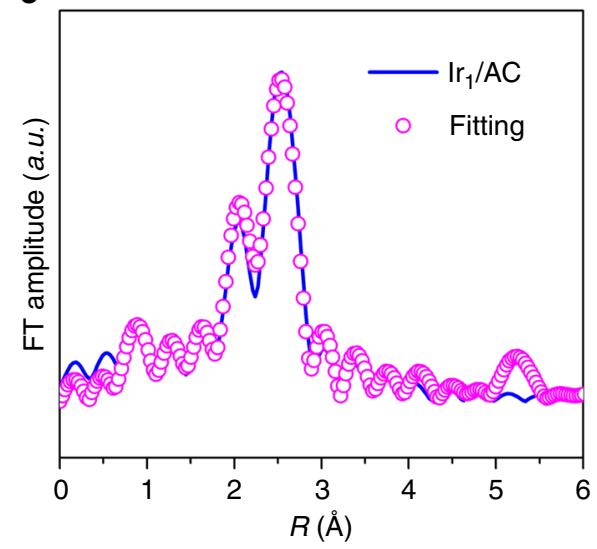

$\mathbf{f}$

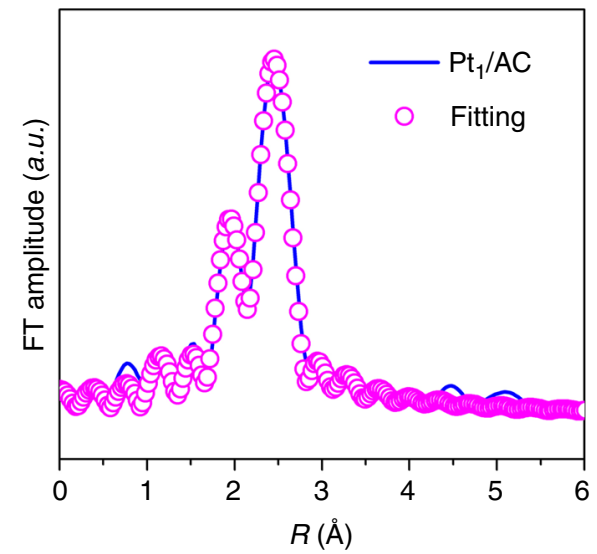

Fig. 5 Atomic dispersion of supported Ir and Pt NPs. a The HRTEM image of Ir/AC; $\mathbf{b}$ the HAADF-STEM image of $\mid \mathrm{r}_{1} / \mathrm{AC}$; $\mathbf{c}$ comparison of the experimental (solid line) and fitted (dashed line) curve of $k^{2}$-weight EXAFS spectra of $\mathrm{Ir}_{1} / \mathrm{AC}$ catalyst. $\mathbf{d}$ The HRTEM image of Pt/AC; e the HAADF-STEM image of Pt 1 / $\mathrm{AC} ; \mathbf{f}$ comparison of the experimental (solid line) and fitted (dashed line) curve of $k^{2}$-weight EXAFS spectra of $\mathrm{Pt}_{1} / \mathrm{AC}_{\mathrm{Catalyst}}$

\section{Methods}

Preparation of catalyst. The coarse coconut shell activated carbon (AC) of 40-60 mesh was washed with deionized water $(353 \mathrm{~K})$ till the electrical conductibility of washings was below $20 \mu \mathrm{s} / \mathrm{cm}$, and afterwards dried at $393 \mathrm{~K}$ for $12 \mathrm{~h}$ (AC-washed). Some of which were calcinated in tubular furnace in a flow of $\mathrm{H}_{2}$ at $1273 \mathrm{~K}$ for $6 \mathrm{~h}$ to eliminate the oxygen-containing group on the surface of AC (AC-treated- $\left.\mathrm{H}_{2}\right)$. Taking $\mathrm{Rh}$ metal as an example, $1.35 \mathrm{~g} \mathrm{RhCl}_{3} \cdot n \mathrm{H}_{2} \mathrm{O}$ with $37 \mathrm{wt} \%$ metal content was dissolved into $15 \mathrm{~mL}$ deionized water, then $10 \mathrm{~g} \mathrm{AC}$-washed or AC-treated- $\mathrm{H}_{2}$ was impregnated, and then stirred continuously till no more bubbles could be discerned, and then dried in $363 \mathrm{~K}$ water bath. The sample was further dried at 393 $\mathrm{K}$ overnight and calcined at $573 \mathrm{~K}$ for $2 \mathrm{~h}$ in a flow of $\mathrm{N}_{2}(100 \mathrm{~mL} / \mathrm{min})$ in quartz tubular reactor. Then the calcined sample was treated with $\mathrm{H}_{2}$ at $573 \mathrm{~K}$ for $2 \mathrm{~h}$ to obtain the fresh $5 \mathrm{wt}$ \% Rh catalyst, which was denoted as Rh/AC. The Rh/AC sample was heated from room temperature to $513 \mathrm{~K}$ in a flow of $\mathrm{N}_{2}$, and held for $20 \mathrm{~min}$, then switched to $\mathrm{CO} / \mathrm{CH}_{3} \mathrm{I}$ mixture (CO passed through a bottle filled with $\mathrm{CH}_{3} \mathrm{I}$ at $298 \mathrm{~K}, 30 \mathrm{~mL} / \mathrm{min}$ ) for $6 \mathrm{~h}$, and cooled to room temperature in a flow of $\mathrm{CO}$ or $\mathrm{N}_{2}$. And the obtained catalyst was denoted as $\mathrm{Rh}_{1} / \mathrm{AC}$. Other samples of $\mathrm{Ru} /$ $\mathrm{AC}, \mathrm{Pd} / \mathrm{AC}, \mathrm{Ag} / \mathrm{AC}, \mathrm{Ir} / \mathrm{AC}$, and $\mathrm{Pt} / \mathrm{AC}$ with $5 \mathrm{wt} \%$ metal loading were prepared and dispersed to $\mathrm{Ru}_{1} / \mathrm{AC}, \mathrm{Pd}_{1} / \mathrm{AC}, \mathrm{Ag}_{1} / \mathrm{AC}, \mathrm{Ir}_{1} / \mathrm{AC}$, and $\mathrm{Pt}_{1} / \mathrm{AC}$ as the similar procedures of that of $\mathrm{Rh} / \mathrm{AC}$ and $\mathrm{Rh}_{1} / \mathrm{AC}$, except that the $\mathrm{Ir} / \mathrm{AC}$ sample was obtained by the reduction of syngas at $513 \mathrm{~K}$ for $2 \mathrm{~h}\left(\mathrm{CO} / \mathrm{H}_{2}=4\right.$ volume ratio).

Catalyst characterization. Inductively coupled plasma optical emission spectrometer (ICP-OES) measurement was performed with PerkinElmer ICP-OES 7300DV. The mixture solution of $\mathrm{H}_{2} \mathrm{O}_{2}$ and concentrated $\mathrm{HNO}_{3}$ was employed to dissolve the samples at high temperature and pressure in a sealed test bottle, and then aquaregia $\left(\mathrm{HCl} / \mathrm{HNO}_{3}=3 / 1 \mathrm{vol}\right)$ was added in to dissolve the residues.

$\mathrm{X}$-ray diffraction (XRD) measurement was performed using PANalytical X'Pert Pro X-ray diffractometer with Cu Ka X-ray source at a wavelength of $1.5045 \AA$. Xray photoelectron spectroscopy (XPS) characterization was performed using a

Thermo fisher ESCALAB 250Xi, which is equipped with an $\mathrm{Al} \mathrm{Ka}$ radiation X-ray source.

The high-resolution transmission electron microscope (HR-TEM) images of the samples were obtained with JEM-2100 equipped with energy dispersive spectrometer (EDS). Particle size distribution was evaluated based on the statistic result with the soft aid of Nano Measure from HRTEM images. The aberrationcorrected high-angle annular dark-field scanning transmission electron microscopy (HAADF-STEM) images were also gained using a JEM-ARM200F STEM/TEM instrument with a CEOS probe corrector working at $200 \mathrm{kV}$ to guarantee a resolution of $0.08 \mathrm{~nm}$.

The obtained Rh HSMSC cooled in a flow of CO was characterized by temperature-programmed desorption-mass spectrum (TPD-MS) using AMI 300 instrument. The $200 \mathrm{mg}$ sample was first pretreated at $323 \mathrm{~K}$ for $1 \mathrm{~h}$ in a flow of helium $(30 \mathrm{~mL} / \mathrm{min})$ to remove species of physical adsorption. After the stabilization of baseline, TPD data were collected using a thermal conductivity detector (TCD) and mass spectrometer at a heating rate of $10 \mathrm{~K} / \mathrm{min}$ to $1273 \mathrm{~K}$. For quantification of total desorbed CO, the peak area of TCD signal was calibrated with pulsed sampling using standard gas of $10 \% \mathrm{CO} / \mathrm{He}$.

Attenuated total reflectance Fourier transform infrared (ATR-FRIR)

experiments were performed by depositing the fine powder of catalyst on top of a Ge internal reflection element (IRE). The spectra were recorded between 800 and $4000 \mathrm{~cm}^{-1}$ by averaging 128 scans at a resolution of $4 \mathrm{~cm}^{-1}$.

Extended X-ray absorption fine structure (EXAFS) data of Rh K-edge were collected at beamline BL14W1 of Shanghai Synchrotron Radiation Facility (SSRF). The data were recorded in the fluorescence mode equipped with Electro-Lyte detector. The original EXAFS data were analyzed by the Demeter software package. Fourier transformation was applied to process the $\mathrm{k}^{3}$-weighted raw data. The theoretical scattering amplitude and phase-shift functions of all the paths for fitting the EXAFS data were calculated with FEFF6 code. The $\mathrm{Rh}_{3}$-edge X-ray adsorption near-edge structure (XANES) spectra were tested at the 4B7A beamline of the Beijing Synchrotron Radiation Facility (BSRF), China, in total electron yield (TEY) mode, where the sample drain current was collected under pressure smaller than $5 \times 10^{-8} \mathrm{~Pa}$.

Solid-state ${ }^{13} \mathrm{C}$ magic angle spinning nuclear magnetic resonance spectroscopy (MAS NMR) experiment was operated on a VARIAN infinity plus spectrometer equipped with a $2.5-\mathrm{mm}$ probe at a frequency of $161.8 \mathrm{MHz}$.

Laser desorption ionization/time of flight mass spectrometer (LDI/TOF-MS) experiments of Rh HSMSC were conducted to detect the laser dissociative fragment of Rh HSMSC and further identify its single complex structure, and the in-situ formed short-lived radical species were also detected with LDI/TOF-MS. The powder of Rh HSMSC sample was attached to an aluminum groove holder of 
$8 \mathrm{~mm} \times 3 \mathrm{~mm}$ in the vacuum chamber. Laser light of $266 \mathrm{~nm}$ was slightly focused on the sample. The skimmed Ar atom beam passed up the sample surface with a distance of $\sim 1 \mathrm{~mm}$ to carry the dissociated and sputtering substance to the detecting chamber. For the in-situ experiment, the $\mathrm{Rh} / \mathrm{AC}$ sample was placed in the back-end of quartz tube reactor. The back-end was designed as an inverted conic shape which has a very small hole in the conic apex to diffuse reactant and radical species into detecting chamber. After introducing $\mathrm{CO} / \mathrm{CH}_{3} \mathrm{I}$ into the reactor, the pressure of the reactor and chamber was kept at $10^{-2}$ and $10^{-7}$ Torr. $\mathrm{CH}_{3} \mathrm{I}$ steam was carried in with $\mathrm{CO}$ at ambient temperature. Then the $\mathrm{Rh} / \mathrm{AC}$ sample was heated by electrical resistance trace heating with a heating rate of $4.0 \mathrm{~K} / \mathrm{min}$ from room temperature to desired temperature in a flow of $\mathrm{CO} / \mathrm{CH}_{3} \mathrm{I}$ mixture $(\mathrm{CO}$ passed through a bottle filled with $\mathrm{CH}_{3} \mathrm{I}$ at $298 \mathrm{~K}, 1 \mathrm{~mL} / \mathrm{min}$ ). The species in detecting chamber would be ionized by $118 \mathrm{~nm}$ radiation. The resultant ions were analyzed by time of flight mass spectrometry equipped with a microchannel plate detector.

Density function theory calculation. The molecule structures were optimized by the density function theory (DFT) calculation using Gaussian 09 package under gasphase condition ${ }^{40}$. The generalized gradient approximation (GGA) of the exchange and correlation function was calculated using B3LYP method ${ }^{41}$ with grime dispersion correction $(\mathrm{GD} 3)^{42}$. We adapted a more accurate basis set, def2-TZVPD ${ }^{43}$ for $\mathrm{Rh}$ and $\mathrm{I}$ atoms and $6-31+\mathrm{G}^{* *}$ for $\mathrm{C}, \mathrm{H}$, and $\mathrm{O}$ atoms. The stable geometries of all $\mathrm{Rh}$ complexes were verified by frequency calculations with no imaginary frequencies. The molecule orbitals (MO) analysis was based on the same basis which produces a small variation of MO. The natural bond order analysis also was performed both with B3LYP function and with 6-311 $++\mathrm{G}^{* *}$ for $\mathrm{C}, \mathrm{H}$, and $\mathrm{O}$ atoms. In this study, the unit of molecule bond length or distance between atoms is angstrom.

In order to reasonably show the role and function of AC in our study system, the carbonyl models of AC have been established and optimized using the B3LYP method. For purpose of studying the dispersion behavior of $\mathrm{Rh}$ atom from the surface of Rh NPs, the geometric structures and the energies were calculated with Vienna ab initio simulation package (VASP) ${ }^{44-47}$. The Perdew-Burke-Ernzerhof $(\mathrm{PBE})$ was used as the exchange correlation function ${ }^{48}$. The cut off kinetic energy was set to $420 \mathrm{eV}$. Brillouin zone integration was approximated using the Monkhorst-Pack $k$-points method ${ }^{49}$ and set as $1 \times 1 \times 1$. The structure models were created with the isolated systems and the side length of simulation box was selected with $36 \AA$ to eliminate the size error. Geometries were optimized until the energy was converged to $1.0 \times 10^{-4} \mathrm{eV} /$ atom and the force was converged to $0.05 \mathrm{eV} / \AA$. Here, it is need to note that the Rh1 atom was randomly selected on the surface of $\mathrm{Rh}$ NPs to clearly show the bond changes between $\mathrm{Rh}_{1}$ atom and neighbor nine $\mathrm{Rh}$ atoms. To understand and account for dispersion effect in the Rh NPs dispersion process, the energy corrections of van der Waals (vdW) were calculated using the semiempirical approach proposed by Grimme ${ }^{50}$ (DFT-D2 method) in conjunction with the PBE functional. All DFT-D2 geometry optimizations of Rh NPs complexes were performed starting from the PBE optimized structures without $\mathrm{vdW}$ correction.

\section{Data availability}

The data that support the findings in this study are in the published article and/or its Supplementary Information files. The data sources are deposited on the generalist repository of figshare, and the accession code is https://doi.org/10.6084/m9. figshare.9948161. The whole datasets are available from the corresponding author on reasonable request. The source data underlying Figs. 1-5 and Supplementary Figs. 1-21, and Supplementary Tables 1-6 are provided as a Source Data file.

Received: 11 March 2019; Accepted: 10 October 2019;

Published online: 21 November 2019

\section{References}

1. Jackson, S. D. Processes occurring during deactivation and regeneration of metal and metal oxide catalysts. Chem. Eng. J. 120, 119-125 (2006).

2. Voorhees, P. W. The theory of Ostwald ripening. J. Stat. Phys. 38, 231-252 (1985).

3. Challa, S. R. et al. Relating rates of catalyst sintering to the disappearance of individual nanoparticles during ostwald ripening. J. Am. Chem. Soc. 133, 20672-20675 (2011).

4. Hansen, T. W., Delariva, A. T., Challa, S. R. \& Datye, A. K. Sintering of catalytic nanoparticles: particle migration or ostwald ripening? Acc. Chem. Res. 46, 1720-1730 (2013).

5. Ouyang, R. H., Liu, J. X. \& Li, W. X. Atomistic theory of Ostwald Ripening and disintegration of supported metal particles under reaction conditions. J. Am. Chem. Soc. 135, 1760-1771 (2013).

6. Hu, S. L. \& Li, W. X. Influence of particle size distribution on lifetime and thermal stability of ostwald ripening of supported particles. ChemCatChem 10, 2900-2907 (2018).
7. Suzuki, A. T. et al. Time scale and elementary steps of CO-induced disintegration of surface Rhodium clusters. Angew. Chem. Int. Ed. 42, 4795-4799 (2003).

8. Parkinson, G. S. et al. Carbon monoxide-induced adatom sintering in a Pd$\mathrm{Fe}_{3} \mathrm{O}_{4}$ model catalyst. Nat. Mater. 12, 724-728 (2013).

9. Morgan, K., Goguet, A. \& Hardacre, C. Metal redispersion strategies for recycling of supported metal catalysts:a perspective. ACS Catal. 5, 3430-3445 (2015).

10. Chong, F. K., Anderson, J. A. \& Rochester, C. H. Effects of oxidation/ reduction and oxychlorination/reduction cycles on $\mathrm{CO}$ adsorption by Pt-Re/ $\mathrm{Al}_{2} \mathrm{O}_{3}$ catalysts. J. Catal. 190, 327-337 (2000).

11. Nagai, Y. et al. In situ redispersion of platinum autoexhaust catalysts: an online approach to increasing catalyst lifetimes? Angew. Chem. Int. Ed. 47, 9303-9306 (2008).

12. Birgersson, $\mathrm{H}$. et al. An investigation of a new regeneration method of commercial aged three-way catalysts. Appl. Catal. B 65, 93-100 (2006).

13. Lambrou, P. S., Polychronopoulou, K., Petallidou, K. C. \& Efstathiou, A. M Oxy-chlorination as an effective treatment of aged $\mathrm{Pd} / \mathrm{CeO}_{2}-\mathrm{Al}_{2} \mathrm{O}_{3}$ catalysts for Pd redispersion. Appl. Catal. B 111, 349-359 (2012).

14. Goguet, A. et al. Increased dispersion of supported gold during methanol carbonylation conditions. J. Am. Chem. Soc. 131, 6973-6975 (2009).

15. Sa, J. et al. Influence of methyl halide treatment on gold nanoparticles supported on Activated Carbon. Angew. Chem. Int. Ed. 50, 8912-8916 (2011).

16. Sa, J. et al. Redispersion of gold supported on oxides. ACS Catal. 2, 552-560 (2012).

17. Duan, X. P. et al. Size controllable redispersion of sintered Au nanoparticles by using iodohydrocarbon and its implications. Chem. Sci. 7, 3181-3187 (2016).

18. Morgan, K. et al. Application of halohydrocarbons for the re-dispersion of gold particles. Catal. Sci. Technol. 4, 729-737 (2014).

19. Wei, S. J. et al. Direct observation of noble metal nanoparticles transformation to thermally stable single atoms. Nat. Nanotechnol. 13, 856-861 (2018).

20. Qu, Y. T. et al. Direct transformation of bulk copper into copper single sites via emitting and trapping of atoms. Nat. Catal. 1, 781-786 (2018).

21. Huang, Z. W. et al. Catalytically active single-atom sites fabricated from silver particles. Angew. Chem. Int. Ed. 51, 4198-4203 (2012).

22. Cui, X. J. et al. Bridging homogenous and heterogeneous catalysis by heterogeneous single-metal-site catalysts. Nat. Catal. 1, 385-397 (2018).

23. Gates, B. C., Flytzani-Stephanopoulos, M., Dixon, D. A. \& Katz, A. Atomically dispersed supported metal catalysts: perspectives and suggestions for future research. Catal. Sci. Technol. 7, 4259-4275 (2017).

24. Babucci, M. et al. Controlling catalytic activity and selectivity for partial hydrogenation by tuning the environment around active sites in iridium complexes bonded to supports. Chem. Sci. 10, 2623-2632 (2019).

25. Serna, P., Yardimci, D., Kistler, J. D. \& Gates, B. C. Formation of supported rhodium clusters from mononuclear rhodium complexes controlled by the support and ligands on rhodium. Phys. Chem. Chem. Phys. 16, 1262-1270 (2014).

26. Yang, D. et al. Metal-organic framework nodes as nearly ideal supports for molecular catalysts: NU-1000-and UiO-66-supported iridium complexes. J. Am. Chem. Soc. 137, 7391-7396 (2015).

27. Serna, P. \& Gates, B. C. Molecular metal catalysts on supports: organometallic chemistry meets surface science. Acc. Chem. Res. 47, 2612-2620 (2014).

28. Aljuhani, M. A. et al. Imine metathesis catalyzed by a silica-supported hafnium imido complex. ACS Catal. 8, 9440-9446 (2018).

29. Xiao, M. L. et al. Identification of binuclear $\mathrm{Co}_{2} \mathrm{~N}_{5}$ active sites for oxygen reduction reaction with more than one magnitude higher activity than single atom $\mathrm{CoN}_{4}$ site. Nano Energy 46, 396-403 (2018).

30. Funke, H., Scheinost, A. C. \& Chukalina, M. Wavelet analysis of extended $\mathrm{x}$ ray absorption fine structure data. Phys. Rev. B. 7109, 4110-4110 (2005).

31. Forster, D. Mechanistic pathways in the catalytic carbonylation of methanol by Rhodium and Iridium complexes. Adv. Organomet. Chem. 17, 255-267 (1979).

32. Henderson, M. A., Mitchell, G. E. \& White, J. M. The chemisorption of methyl halides (Cl, Br, and I) on Pt (111). Surf. Sci. 184, L325-L331 (1987).

33. Wettergren, K. et al. High sintering resistance of size-selected platinum cluster catalysts by suppressed Ostwald Ripening. Nano Lett. 14, 5803-5809 (2014).

34. Su, Y. Q., Liu, J. X., Filot, I. A. W. \& Hensen, E. J. M. Theoretical study of ripening mechanisms of Pd clusters on ceria. Chem. Mater. 29, 9456-9946 (2017).

35. Figueiredo, J. L., Pereira, M. F. R., Freitas, M. M. A. \& Orfao, J. J. M. Modification of the surface chemistry of activated carbons. Carbon 37 1379-1389 (1999).

36. Yan, H. et al. Single-atom $\mathrm{Pd}_{1} /$ graphene catalyst achieved by atomic layer deposition: remarkable performance in selective hydrogenation of 1,3butadiene. J. Am. Chem. Soc. 137, 10484-10487 (2015).

37. Yan, H. et al. Bottom-up precise synthesis of stable platinum dimers on graphene. Nat. Commun. 8, 1070 (2017). 
38. Hensley, A. J. R. et al. Mechanistic understanding of methanol carbonylation: Interfacing homogeneous and heterogeneous catalysis via carbon supported Ir-La. J. Catal. 361, 414-422 (2018).

39. Wang, J. et al. Formation, migration, and reactivity of Au-CO complexes on gold surfaces. J. Am. Chem. Soc. 138, 1518-1526 (2016).

40. M. J. Frisch, et al. Gaussian 09, Revision A.02 (Gaussian, Inc., Wallingford, CT, 2009).

41. Zhao, Y. \& Truhlar, D. G. The M06 suite of density functionals for main group thermochemistry, thermochemical kinetics, noncovalent interactions, excited states, and transition elements: two new functionals and systematic testing of four M06-class functionals and 12 other functionals. Theor. Chem. Acc. 120, 215-241 (2008).

42. Grimme, S., Antony, J., Ehrlich, S. \& Krieg, H. A consistent and accurate ab initio parameterization of density functional dispersion correction (DFT-D) for the 94 elements H-Pu. J. Chem. Phys. 132, 154104 (2010).

43. Feller, D. The role of databases in support of computational chemistry calculations. J. Comp. Chem. 17, 1571-1586 (1996).

44. Kresse, G. \& Furthmuller, J. Efficient iterative schemes for ab initio totalenergy calculations using a plane-wave basis set. Phys. Rev. B 54, 11169-11186 (1996).

45. Kresse, G. \& Hafner, J. Ab initio molecular-dynamics for liquid-metals. Phys. Rev. B 47, 558-561 (1993).

46. Kresse, G. \& Hafner, J. Ab-initio molecular-dynamics simulation of the liquidmetal amorphous-semiconductor transition in germanium. Phys. Rev. B 49, 14251-14269 (1994).

47. Kresse, G. \& Furthmuller, J. Efficiency of ab-initio total energy calculations for metals and semiconductors using a plane-wave basis set. Phys. Rev. B 6, 15-50 (1996).

48. Perdew, J. P., Burke, K. \& Ernzerhof, M. Generalized gradient approximation made simple. Phys. Rev. Lett. 77, 3865-3868 (1996).

49. Monkhorst, H. J. \& Pack, J. D. Special points for brillouin-zone integrations. Phys. Rev. B 13, 5188-5192 (1976).

50. Grimme, S. Semiempirical GGA-type density functional constructed with a long-range dispersion correction. J. Comput. Chem. 27, 1787-1799 (2006).

\section{Acknowledgements}

The financial support of this work by National Key R\&D Program of China (2017YFB0602203), the Strategic Priority Research Program of the Chinese Academy of Sciences, Grant No. XDA21020300 and Grant No XDB17020400, Natural Science Foundation of Zhejiang Province, China (LY18B060007). We thank Prof. Z. Conrad Zhang for his helpful discussion.

\section{Author contributions}

S.F. prepared and characterized the samples, and performed catalytic evaluation. X.S designed the experiments and drafted the manuscript. L.Y. helped with the HAADFSTEM and EXAFS characterizations. Y.L. and Z.J. were responsible for the EXAFS measurement and interpretation of results. X.L. was responsible for the DFT calculations S.L., W.D., and X.Y. helped with the laser desorption ionization/time of flight mass spectrometer experiments. Y.D. conceived the research, designed the experiments, and drafted the manuscript. All the authors discussed the results and participated in writing the manuscript.

\section{Competing interests}

The authors declare no competing interests.

\section{Additional information}

Supplementary information is available for this paper at https://doi.org/10.1038/s41467019-12965-1.

Correspondence and requests for materials should be addressed to X.S., X.L., Z.J. or Y.D.

Peer review information Nature Communications thanks the anonymous reviewers for their contribution to the peer review of this work. Peer reviewer reports are available.

Reprints and permission information is available at http://www.nature.com/reprints

Publisher's note Springer Nature remains neutral with regard to jurisdictional claims in published maps and institutional affiliations.

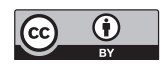

Open Access This article is licensed under a Creative Commons Attribution 4.0 International License, which permits use, sharing, adaptation, distribution and reproduction in any medium or format, as long as you give appropriate credit to the original author(s) and the source, provide a link to the Creative Commons license, and indicate if changes were made. The images or other third party material in this article are included in the article's Creative Commons license, unless indicated otherwise in a credit line to the material. If material is not included in the article's Creative Commons license and your intended use is not permitted by statutory regulation or exceeds the permitted use, you will need to obtain permission directly from the copyright holder. To view a copy of this license, visit http://creativecommons.org/ licenses/by/4.0/.

(C) The Author(s) 2019 